\title{
HUMAN RED CELL GPT POLYMORPHISM: COMPARISON OF THE ACTIVITIES AMONG DIFFERENT PHENOTYPES USING PYRUVATE AND L-GLUTAMATE AS SUBSTRATES
}

\author{
Shintaroh UeDA, * Makoto UCHIKawa,** and Keiichi Omoto* \\ *Department of Anthropology, Faculty of Science, The University of Tokyo, Tokyo \\ **Blood Transfusion Service, Tokyo University Hospital, Tokyo
}

\begin{abstract}
Summary A convenient method for measuring human red cell GPT activity using pyruvate and L-glutamate as substrates (the reverse reaction) was described and the activities among different GPT phenotypes were compared. It was found that the mean activity for GPT 1 phenotype was higher than that for GPT 2, while that for GPT 2-1 fell between these two levels. These results accorded with the finding known as to the forward reaction.
\end{abstract}

\section{INTRODUCTION}

Glutamic-pyruvic transaminase (GPT), also known well as alanine aminotransferase [EC 2.6.1.2], catalyzes reversibly the following reaction.

L-alanine $+\alpha$-ketoglutarate $\rightleftharpoons$ pyruvate $+\mathrm{L}$-glutamate

The conversion of L-alanine and $\alpha$-ketoglutarate to pyruvate and L-glutamate is referred to as the forward reaction. This enzyme exsists in both cytoplasmic (soluble) and mitochondrial fractions. An equilibrium constant of 1.6 was reported at $\mathrm{pH} 7.3$ and $37^{\circ} \mathrm{C}$ in the direction of L-alanine and $\alpha$-ketoglutarate formation with rat liver soluble GPT (Segal et al., 1962).

Genetic polymorphism of soluble GPT was reported in human red blood cells (Chen and Giblett, 1971). Three common phenotypes were found by starch gel electrophoresis, representing the two homozygotes and a heterozygote of a pair of autosomal, codominant alleles, $G p t^{1}$ and $G p t^{2}$. In addition to these common alleles, six rare alleles, $G p t^{3}, G p t^{4}, G p t^{5}, G p t^{6}$ (Chen et al., 1972), Gpt ${ }^{7}$ (Olaisen, 1973a), $G p t^{8}$ (Santachiara Benerecetti et al., 1975), and silent alleles (Olaisen, 1973b; Spielman et al., 1973) have thus far been found.

The differences in the average activity between the three common phenotypes have been reported (Chen et al., 1972; Welch, 1972; Olaisen, 1973b; Welch, 1975; Nishigaki et al., 1978). The mean activity of GPT 1 is about 1.5 to 3.5 times higher than that of GPT 2, and that of GPT 2-1 is nearly intermediate. These reports, 
however, have thus far been limited to the reaction using L-alanine and $\alpha$-ketoglutarate as substrates although this enzyme catalyzes the reversible reaction.

In this report, we describe a convenient method for measuring red cell GPT activity using pyruvate and L-glutamate as substrates and report the results as to whether there is a similar activity difference between phenotypes as in the forward reaction.

\section{MATERIALS AND METHODS}

Fresh blood samples collected in ACD solution were obtained from 164 unrelated blood donors in Tokyo. For the measurement of enzyme activity, hemolysates were prepared by adding one volume of cold distilled water to one volume of washed, packed red cells (Chen et al., 1971).

Red cell GPT activity was determined by measuring the amount of $\alpha$-ketoglutarate formed in the reaction, using a selective microphotometric method described by Hamada and Ohkura (1976), with modifications. The measurement of $\alpha$-ketoglutarate was carried out on the basis of a colour reaction with diazotized sulfamethizole in a sodium hydroxide solution in the presence of sodium sulfite and sodium hypophosphite. The following reagents were used;

(1) Substrate solution: $0.1 \mathrm{M} \mathrm{N}$-2-hydroxyethylpiperazine-N'-2-ethanesulfonic acid (Hepes), $0.1 \mathrm{M}$ L-glutamate (sodium salt), and $0.005 \mathrm{M}$ pyruvate (sodium salt), $\mathrm{pH} 7.5$.

(2) Diazotized sulfamethizole solution: To $100 \mathrm{ml}$ of $0.9 \%$ (w/v) $\mathrm{N}^{1}-(5-$ methyl-1, 3,4-thiadiazol-2-yl)-sulfanilamide (sulfamethizole) in $2 \mathrm{~N} \mathrm{HCl}$ solution, $10 \mathrm{ml}$ of freshly prepared $2.5 \%(\mathrm{w} / \mathrm{v})$ sodium nitrite solution was added.

(3) Alkaline solution: $1 \%(\mathrm{w} / \mathrm{v})$ sodium sulfite and $1.25 \%(\mathrm{w} / \mathrm{v})$ sodium hypophosphite in $9 \%(\mathrm{w} / \mathrm{v})$ sodium hydroxide.

To $0.5 \mathrm{ml}$ of the substrate solution, $20 \mu \mathrm{l}$ of the hemolysate was added and incubated for $60 \mathrm{~min}$ at $37^{\circ} \mathrm{C}$ before transamination was terminated by the addition of $1.0 \mathrm{ml}$ of the diazotized sulfamethizole solution in an ice-water bath. Then, $8.0 \mathrm{ml}$ of the alkaline solution was added. After incubation for $60 \mathrm{~min}$ at $37^{\circ} \mathrm{C}$, the reaction mixture was cooled in an ice-water bath and the absorbance at $540 \mathrm{~nm}$ was measured within 30min using a Hitachi 101 spectrophotometer. The blank was prepared in the same way, omitting the first incubation.

This method is convenient for the measurement of red cell GPT activity in genetic studies from the following reasons. (1) The amount of sample needed is much smaller than that in the dinitrophenylhydrazine method (Chen et al., 1972; Olaisen, 1973b; Nishigaki et al., 1978). (2) The assay does not need enzyme and coenzyme in the reaction mixture, and can be carried out with a relatively simple spectrophotometer, compared with the LDH coupling method (Welch, 1972, 1975).

One unit of enzyme activity was defined as the amount of enzyme which produced $1 \mu$ mole of $\alpha$-ketoglutarate per minute under this condition. The amount of 
hemoglobin in the hemolysate was estimated by the cyanmethemoglobin method and the red cell enzyme activity was expressed in units per gram of hemoglobin.

Electrophoresis, specific staining, and typing were carried out as described by Chen and Giblett (1971) with modifications: Hemolysates were prepared by freezing and thawing red cells. Horizonal starch gel electrophoresis was carried out for $18 \mathrm{hr}$ at $4{ }^{\circ} \mathrm{C}$ at $6-8 \mathrm{~V} / \mathrm{cm}$, using $0.1 \mathrm{M}$ Tris- $0.1 \mathrm{M}$ maleate-0.01M EDTA-0.01M $\mathrm{MgCl}_{2}(\mathrm{pH} 7.4)$ (Spencer et al., 1964), as the bridge buffer. The gels were prepared containing $11-12 \%$ starch (Connaught Medical Research Laboratories) in a 1 in 20 dilution of the bridge buffer. The staining mixture consisted of $0.28 \mathrm{M} \mathrm{L}$-alanine, $0.03 \mathrm{M} \alpha$-ketoglutarate (free acid), $1.3 \mathrm{~mm} \mathrm{NADH}$, and approximately 20 units $/ \mathrm{ml}$ lactate dehydrogenase in $0.3 \mathrm{M}$ Tris- $\mathrm{HCl}(\mathrm{pH} 8.0)$.

\section{RESULTS AND DISCUSSION}

GPT typing by starch gel electrophoresis of 164 samples revealed the following phenotypic distribution: GPT 1 to $50(30 \%)$, GPT $2-176(46 \%)$, GPT $237(23 \%)$. A single sample was found to be a rare phenotype GPT 3-1. The distribution of GPT activity measured for the reverse reaction in the three common phenotypes is shown in Fig. 1. It was found that the range of GPT 1 activity was greater than that of GPT 2 in the reverse reaction as it is the case in the forward reaction. However, there was no indication from these data of a genetic heterogeneity among samples of

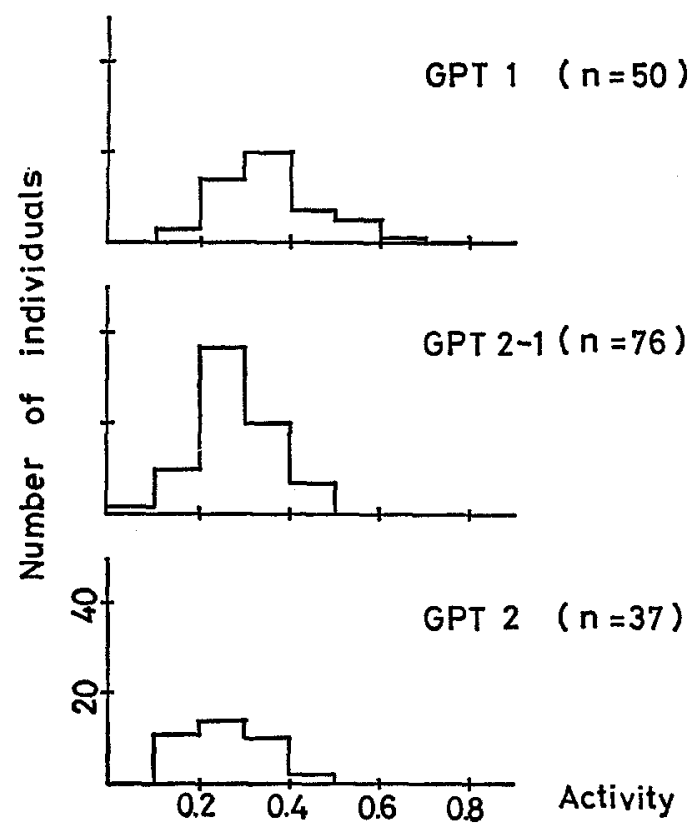

Fig. 1. Distribution of red cell GPT activity in the three common phenotypes. 
Table 1. The mean activity of the three common GPT phenotypes and the ratio of mean activity of GPT 1 to that of GPT 2.

\begin{tabular}{|c|c|c|c|c|c|c|}
\hline & & \multicolumn{5}{|c|}{ Mean activity } \\
\hline & & $N$ & GPT 1 & GPT 2-1 & GPT 2 & GPT 1/GPT 2 \\
\hline \multicolumn{7}{|l|}{ Forward reaction } \\
\hline Chen et al. & (1972) & 275 & 4.17 & $\begin{array}{c}2.75 \\
(2.92)\end{array}$ & 1.67 & 2.50 \\
\hline Welch & $(1972)$ & 61 & 0.588 & $\begin{array}{c}0.361 \\
(0.380)\end{array}$ & 0.172 & 3.42 \\
\hline Olaisen & (1973b) & 158 & 8.09 & $\begin{array}{l}5.55 \\
(5.74)\end{array}$ & 3.39 & 2.39 \\
\hline Welch & (1975) & 126 & 0.617 & $\begin{array}{c}0.488 \\
(0.512)\end{array}$ & 0.406 & 1.52 \\
\hline Nishigaki et al. & (1978) & 145 & 6.55 & $\begin{array}{c}4.66 \\
(4.67)\end{array}$ & 2.78 & 2.36 \\
\hline \multicolumn{7}{|l|}{ Reverse reaction } \\
\hline Present study & & 164 & 0.356 & $\begin{array}{c}0.281 \\
(0.308)\end{array}$ & 0.259 & 1.37 \\
\hline
\end{tabular}

Enzyme activity according to each reference.

( ): The half value of a simple summation of GPT 1 and GPT 2.

the type GPT 1. The mean activities were found to be 0.356 [units/gHb] for GPT 1 $(\mathrm{n}=50), 0.281$ [units/gHb] for GPT 2-1 $(\mathrm{n}=76)$, and 0.259 [units/gHb] for GPT 2 $(\mathrm{n}=37)$, with standard deviations $0.106,0.086$, and 0.086 , respectively.

The activity of a single sample with a rare variant phenotype GPT 3-1 was found to be 0.417 [units $/ \mathrm{gHb}$ ]. As to the activity of the rare variants, there is a report about GPT 7-1 $(n=2)$ and GPT 7-2 $(n=6)$ for the forward reaction (Olaisen, 1973a). It was suggested that the average activity attributable to the $G p t^{7}$ allele is close to that of the $G p t^{1}$ allele. On the other hand, this case of GPT 3-1 had a relatively high activity. However, the activity of the GPT 3 isozyme in the present sample is weaker than that of the GPT 1 isozyme as judged from the zymogram in accordance with the diagram of GPT phenotypes presented by Chen et al. (1972). More samples are clearly needed to determine the mean activity attributable to the variant $G p t^{3}$ allele.

The data of GPT activity for the three common phenotypes so far reported are shown in Table 1. In the forward reaction, the ratio of mean activity of GPT 1 to that of GPT 2 is about 1.5 to 3.5. On the other hand, it is smaller (1.37) in the reverse reaction. However, the difference in the mean activities between GPT 1 and GPT 2 was statistically significant $(t=4.473, \mathrm{p}<0.005)$. Thus, it is now clear that the mean activity of GPT 1 is higher than that of GPT 2 and that of GPT 2-1 falls between these two levels in the both reactions.

The difference in enzyme activity between phenotypes have not been clarified by the studies of optimal $\mathrm{pH}$, heat stability, and Michaelis constants in the forward 
reaction using partially purified enzyme (Welch, 1975). Homogeneously purified GPT is needed to discuss whether the difference in enzyme activity between phenotypes is due to the qualitative difference (turn-over number) or/and the quantitative difference (absolute quantity of the enzyme).

Acknowledgments The authors are grateful to Dr. S. Horai and Mr. K. Tokunaga for the arrangement of the sample collection and valuable discussion. They also wish to express their gratitude to Dr. O. Takenaka, the University of Kyoto, for his helpful advice and criticism.

\section{REFERENCES}

Chen, S. H. and Giblett, E. R. 1971. Polymorphism of soluble glutamic-pyruvic transaminase: A new genetic marker in man. Science 173: 148-149.

Chen, S. H., Malcolm, L. A., Yoshida, A., and Giblett, E. R. 1971. Phosphoglycerate kinase: An X-linked polymorphism in man. Am. J. Human Genet. 23: 87-91

Chen, S. H., Giblett, E. R., Anderson, J. E., and Fossum, B. L. G. 1972 . Genetics of glutamicpyruvic transaminase: Its inheritance, common and rare variants, population distribution, and differences in catalytic activity. Ann. Human Genet. 35: 401-409.

Hamada, H. and Ohkura, Y. 1976. A new photometric method for the determination of serum glutamic pyruvic transaminase activity using pyruvate and glutamate as substrates. Chem. Pharm. Bull. (Tokyo) 24: 1865-1869.

Nishigaki, I., Suzuki, H., Fujiki, N., and Nakai, T. 1978. Genetic polymorphism of human red cell glutamic-pyruvic transaminase in an isolated community in western Japan. Jap. J. Human Genet. 23: 9-15.

Olaisen, B. 1973a. Two rare GPT phenotypes in a Norwegian family. Evidence of a seventh allele. Humangenetik 19: 289-291.

Olaisen, B. 1973b. Atypical segregation of erythrocyte glutamic-pyruvic transaminase in a Norwegian family. Evidence of a silent allele. Human Hered. 23: 595-602.

Santachiara Benerecetti, A.S., Beretta, M., and Pampiglione, S. 1975. Red cell glutamic-pyruvic transaminase polymorphism in a sample of the Italian population. A new variant allele: GPT. Human Hered. 25: 276-278.

Segal, H. L., Beattie, D. S., and Hopper, S. 1962. Purification and properties of liver glutamicalanine transaminase from normal and corticoid-treated rats. J. Biol. Chem. 237: 1914-1920.

Spencer, N., Hopkinson, D. A., and Harris, H. 1964. Phosphoglucomutase polymorphism in man. Nature 204: 742-745.

Spielman, W., Kühnl, P., Rexrodt, Ch. and Hänsel, G. 1973. Untersuchungen zum GPT System unter besonderer Berücksichtingung des stummen Alleles GPT ${ }^{0}$. Humangenetik 18: 341-348.

Welch, S. G. 1972. Quantitative differences between the human red cell glutamic-pyruvic transaminase phenotypes. Human Hered. 22: 190-197.

Welch, S. 1975. Comparative studies on the human glutamate-pyruvate transaminase phenotypes - GPT 1, GPT 2-1, GPT 2. Humangenetik 30: 237-249. 